\section{Incidence of primary malignant orbital tumours in the Netherlands}

JH Koopman', M van der Heiden-van der Loo², MR van Dijk ${ }^{3}$ and WR Bijlsma ${ }^{4}$

\begin{abstract}
Aims To describe the incidence and relative frequencies of primary malignant orbital tumours in the Netherlands from 1989 to 2006. Methods All registered primary malignant orbital tumours were extracted from the population-based database of the Netherlands Cancer Registry. Age-adjusted incidence of malignant orbital tumours per 10000000 persons per year and the estimated annual percentage change (EAPC) were computed. Results A total of 367 malignant orbital tumours were registered. The average age-adjusted incidence of malignant orbital tumours is 10.9. Lymphoma has a relative frequency of $67 \%$, rhabdomyosarcoma $12 \%$, adenocarcinoma $6 \%$, and adenoid cystic carcinoma $5 \%$. The incidence of primary malignant orbital tumours has been increasing in the Netherlands (EAPC $+\mathbf{2 . 8 \%}$ ).

Conclusion In the Netherlands, lymphoma is the most common primary malignant orbital tumour, followed by rhabdomyosarcoma, adenocarcinoma, and adenoid cystic carcinoma. The relative frequencies of the different histological tumour types are comparable to the frequencies in other parts of the world. The incidence of malignant primary orbital tumours shows a slight increase between 1989 and 2006.

Eye (2011) 25, 461-465; doi:10.1038/eye.2011.9; published online 18 February 2011
\end{abstract}

Keywords: tumour; incidence; cancer; orbit; lacrimal gland

\section{Introduction}

Knowledge of the epidemiology of orbital tumours, can aid the clinician in developing a differential diagnosis when presented with a patient with proptosis or eyelid swelling.
The incidence of malignant tumours of all primary sites is rising, ${ }^{1}$ it is also of interest if orbital tumours show this same rising trend. Only one study reports on the incidence numbers of malignant orbital tumours on a population-based level, this study was conducted in the USA and shows a rising trend. ${ }^{2}$ These data are not available for Europe.

Cancers can show geographical differences in their relative frequencies, for example uveal melanoma is relatively more common in North America and Europe. ${ }^{3,4}$ Geographical differences in orbital tumour frequencies may suggest environmental factors are involved in disease development.

The first goal of this paper is to report on the incidence numbers of malignant orbital tumours in the Netherlands and its development over time, to check for possible trends in time. The second goal of our study is to determine the relative frequencies of different histological subtypes of malignant orbital tumours and compare these with other parts of the world, to see if there are any geographical differences.

\section{Materials and methods}

All primary malignant orbital tumours were selected from the Netherlands Cancer Registry (NCR) database from 1989 to 2006, limited to residents of the Netherlands.

Orbit is defined as the tissues within the orbital cavity posterior to the orbital septum (including the lacrimal gland). In this study, tumours directly extending from within the globe, the eyelid skin, the conjunctiva, the accessory lacrimal glands or the paranasal sinuses to the orbit or distant metastasis to the orbit are excluded.

The NCR records all cancer cases from 1989 onwards. New cancer cases are detected through a nation-wide network and registry of
'Department of Ophthalmology, University Medical Center Utrecht, Utrecht, The Netherlands

${ }^{2}$ Comprehensive Cancer Center Middle Netherlands, Utrecht, The Netherlands

${ }^{3}$ Department of Pathology, University Medical Center Utrecht, Utrecht,

The Netherlands

${ }^{4}$ Department of Ophthalmology, University Medical Center Utrecht,

Utrecht, The Netherlands

Correspondence: JH Koopman, Department of Ophthalmology, University Medical Center Nijmegen, Ph van Leydenlaan 15, $6500 \mathrm{HB}$ Nijmegen, The Netherlands Tel: + 003162552 8759; Fax: + 0031243613501 . E-mail: jhkoopman@ hotmail.com

Received: 11 August 2010 Accepted in revised form: 9 December 2010 Published online: 18 February 2011 
histo- and cytopathology (PALGA). ${ }^{5}$ Other sources of cases are a national registry of hospital discharge diagnoses (LMR) and haematological laboratories. Specially trained NCR staff records data of new cancer cases from the medical files in the hospitals. The international classification of diseases for oncology (ICD-O) is used as basis for coding of tumours. ${ }^{6}$

In this study the topographical site codes C69.6 (for orbit) and C69.5 (for tear gland) were used to identify primary malignant orbital tumours. The topographical codes C69.3 (for choroid) and C69.4 (for ciliary body) were used to filter out all uveal tumours. Subsequently, melanomas (morphological codes 8720 through 8790) were selected, to compare the number of uveal melanomas with the number of primary malignant orbital tumours.

Tumour types with closely related morphological characteristics and clinical behaviour, were grouped in consultation with an eye pathologist (MRD).

To calculate the crude incidence, the number of tumours per year was divided by the mid-year population provided by the Netherlands Population Statistics. ${ }^{7}$ To adjust for the demographic factor age, the world standardised ratio (WSR) was calculated. ${ }^{8}$ To reduce short-term fluctuations, we used the centred 3-year moving average of the WSR.
To examine time trends in incidence rates the estimated annual percentage change (EAPC) was calculated for the overall tumour incidence, using the statistical software package Stata (Stata Corporation, College Station, TX, USA). An EAPC with a $P$-value $<0.05$ is considered significant.

This study used anonymised data and was approved by the privacy board of the NCR and is in line with the Dutch privacy regulations and the declaration of Helsinki.

\section{Results}

In the period 1989 to 2006 , a total of 3640 orbital and ocular tumours were registered in the Netherlands. The most frequent tumour encountered was a uveal melanoma ( $n=2193)$. A total number of 367 primary malignant orbital tumours, in 367 patients, were registered. In 89 cases the lacrimal gland was the site of origin and all other tumours $(n=278)$ originated in the orbital soft tissues outside the lacrimal gland (Table 1). Overall the most common tumour type was lymphoma $(67 \%)$, both within the lacrimal gland $(51 \%)$ and in the orbital soft tissues other then the lacrimal gland (72\%). The next most common tumour types were rhabdomyosarcoma (12\%), adenocarcinoma (6\%), and

Table 1 Primary malignant orbital tumours: location, age and gender distribution, and average crude and age standardised incidence rates per 10000000 per year in the Netherlands from 1989 to 2006

\begin{tabular}{|c|c|c|c|c|c|c|c|}
\hline \multirow[t]{2}{*}{ Tumour morphology (ICD-O codes) } & \multirow[t]{2}{*}{$\begin{array}{c}\text { Lacrimal } \\
\text { gland }\end{array}$} & \multirow[t]{2}{*}{$\begin{array}{l}\text { Orbital tissues } \\
\text { outside the } \\
\text { lacrimal } \\
\text { gland }\end{array}$} & \multirow{2}{*}{$\begin{array}{c}\text { Gender } \\
\text { Male: female }\end{array}$} & \multicolumn{2}{|c|}{ Age } & \multirow[t]{2}{*}{$\begin{array}{c}\text { Average } \\
\text { crude } \\
\text { incidence }\end{array}$} & \multirow[t]{2}{*}{$\begin{array}{c}\text { Average world } \\
\text { standardised } \\
\text { rate }\end{array}$} \\
\hline & & & & Median & Min-max & & \\
\hline Neoplasm malignant (8000) & 0 & 3 & $1: 2$ & 77 & $67-90$ & 0.11 & 0.05 \\
\hline Carcinoma others $(8010,8020$, and 8041) & 2 & 1 & $1: 2$ & 66 & $47-75$ & 0.11 & 0.07 \\
\hline Squamous cell carcinomas $(8052,8070$, and 8083$)$ & 5 & 3 & $4: 4$ & 72 & $38-93$ & 0.28 & 0.17 \\
\hline Adenocarcinoma $(8140,8410$, and 8430$)$ & 19 & 3 & $15: 7$ & 69 & $42-83$ & 0.78 & 0.51 \\
\hline Adenoid cystic carcinoma (8200) & 17 & 2 & 9:10 & 46 & $23-75$ & 0.68 & 0.52 \\
\hline Malignant melanoma NOS $(8720)^{a}$ & 1 & 2 & $1: 2$ & 66 & $65-67$ & 0.11 & 0.07 \\
\hline $\begin{array}{l}\text { Fibrohistiocytic sarcomas }(8801,8810,8815,8830 \text {, } \\
\text { and 9755) }\end{array}$ & 0 & 6 & $3: 3$ & 59 & $4-89$ & 0.21 & 0.18 \\
\hline Leiomyosarcoma NOS (8890) ${ }^{a}$ & 0 & 2 & $0: 2$ & 53 & $22-84$ & 0.07 & 0.05 \\
\hline Malignant rhabdoid tumour (8963) & 0 & 1 & $0: 1$ & 0 & $0-0$ & 0.04 & 0.07 \\
\hline Rhabdomyosarcoma (8900, 8910, and 8920) & 0 & 43 & $25: 18$ & 8 & $0-72$ & 1.53 & 2.40 \\
\hline $\begin{array}{l}\text { Chondro-osseous sarcomas }(9180,9181,9220, \\
\text { and 9240) }\end{array}$ & 0 & 5 & $2: 3$ & 53 & $16-67$ & 0.18 & 0.18 \\
\hline Ewing sarcoma (9260) & 0 & 2 & $2: 0$ & 9 & $2-16$ & 0.07 & 0.12 \\
\hline MPNST $(9540 \text { and } 9561)^{b}$ & 0 & 4 & $2: 2$ & 58 & $1-81$ & 0.14 & 0.13 \\
\hline Alveolar soft part sarcoma (9581) & 0 & 1 & $1: 0$ & 19 & 19-19 & 0.07 & 0.12 \\
\hline $\begin{array}{l}\text { Lymphoma (9590, 9591, 9670, 9671, 9673, 9675, } \\
\text { 9680, 9690, 9691, 9695, 9698, 9699, 9702, and 9719) }\end{array}$ & 45 & 200 & 130:115 & 68 & $6-93$ & 8.71 & 5.58 \\
\hline Total & 89 & 278 & 196:171 & 66 & $0-93$ & 13.04 & 10.15 \\
\hline
\end{tabular}

Abbreviation: ICD-O, international classification of disease oncology.

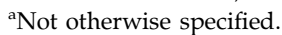

bMalignant peripheral nerve sheath tumours. 
adenoid cystic carcinoma (5\%). All other tumour types had a relative frequency of $2 \%$ or less. The lacrimal gland was the primary site of adenocarcinoma and adenoid cystic carcinoma in 86 and $89 \%$, respectively.

The percentage of male patients was $53 \%(n=196)$ and the median age was 66 years at the time of diagnosis. The patient ages ranged from 0 to 93 years. Two age peaks can be identified (Figure 1), between 0 and 15 years and between 60 and 80 years. In the age group below 15 years $86 \%$ of the tumours was a rhabdomyosarcoma. Between 60 and 80 years, lymphoma (79\%) and carcinoma (14\%) predominated.

The overall average crude incidence was 13 per 10000000 per year and the WSR was 10 per 10000000 per year (Table 1). Figure 2 shows the WSR for males and females over time. The EAPC for the WSR in the years 1989 to 2006 was $+2.5 \%(P=0.089)$ for men, $+3.0 \%$

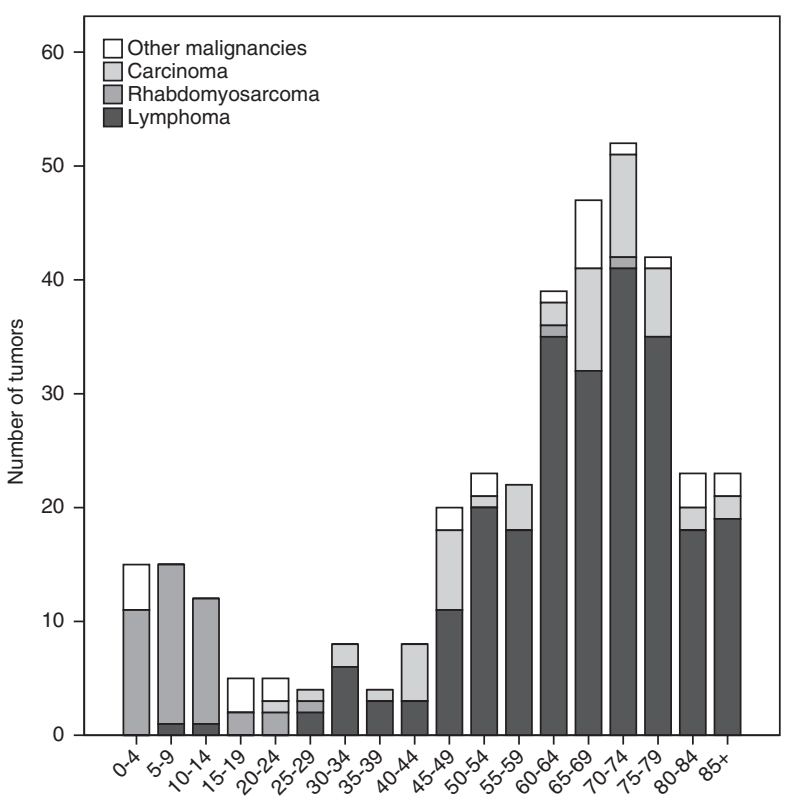

Figure 1 Age distribution among the three largest histological subgroups and the remaining primary malignant orbital tumours in the Netherlands from 1989 to 2006.

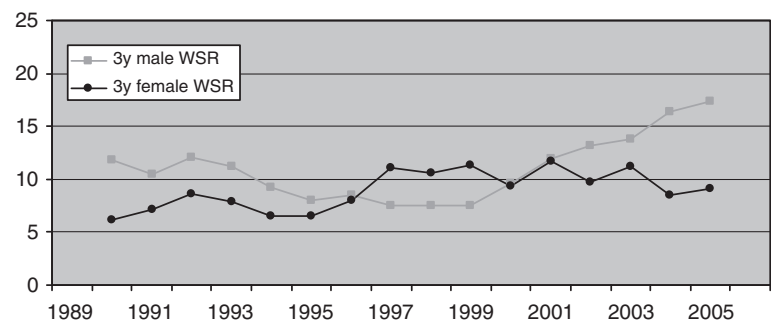

Figure 2 The course of primary malignant orbital tumour incidence (centred 3-year moving average) standardised to the world population (WSR) from 1989 to 2006.
$(P=0.006)$ for women, and $+2.8 \%(P=0.001)$ for men and women combined.

\section{Discussion}

The most common malignant orbital tumours found in the population of the Netherlands from 1989 to 2006 were lymphoma followed by rhabdomyosarcoma, adenocarcinoma, and adenoid cystic carcinoma. In the 18-year study period, the number of orbital tumours was small compared with the number of uveal melanoma.

Other studies based on cancer registries, performed in Asia and in the USA, show similar relative frequencies of tumour types (Table 2) and all conclude that the predominant primary malignant orbital tumour is lymphoma. ${ }^{2-4}$

The third and fourth most common tumours, adenocarcinomas and adenoid cystic carcinomas, also compare with the rest of the world. ${ }^{2-4}$ This suggests there are no geographical differences in relative frequencies of orbital tumour types (see Table 2), as there are for uveal melanoma and retinoblastoma. ${ }^{2-4}$

Other studies report squamous cell carcinoma (SCC) as the second most common tumour type, instead of rhabdomyosarcoma in our study. ${ }^{2-4}$ As is mentioned by Margo and Mulla, ${ }^{2}$ this high frequency of SCC's is probably in part the result of coding errors. Their explanation is that it is more likely that these tumours represent a secondary growth from structures surrounding the orbit, such as the eyelids and the paranasal sinuses. Our study includes eight SCC's, two Ewing sarcomas, and three melanomas that are rare primary tumours of the orbit. In retrospect, some of these registrations may represent coding errors but overall the number of coding errors is still very low.

The average crude incidence of orbital tumours in the Netherlands is lower compared with the incidence in Florida (13 vs 20 per 10000000 persons per year). As age is one of the most important factors in developing cancer, we decided to correct for age using the WSR. The average

Table 2 Comparison of relative frequencies of primary malignant tumour types in the Netherlands with other parts of the world

\begin{tabular}{lcc}
\hline Tumour morphology & $\begin{array}{c}\text { The } \\
\text { Netherlands (\%) }\end{array}$ & $\begin{array}{c}\text { Other } \\
\text { countries (\%) }\end{array}$ \\
\hline Lymphoma & 67 & $35-55$ \\
Squamous cell carcinoma & 2 & $10-11$ \\
Rhabdomyosarcoma & 12 & $5-10$ \\
Adenocarcinoma & 6 & $2-8$ \\
Adenoid cystic carcinoma & 5 & $4-10$ \\
\hline
\end{tabular}

aUS, Singapore, and Taiwan. 
world standardised incidence in our study is 10 , which is lower then the average crude incidence. This can be explained by the relatively older population in the Netherlands as compared with the world population and the fact that most orbital tumours occur at older ages.

The majority of the epidemiological information on primary malignant orbital tumours is based on studies performed in tertiary care centres. ${ }^{9-14}$ These tertiary centres can have a special interest or expertise in specific tumour types. This inevitably leads to an overrepresentation of these tumour types and therefore the relative frequencies cannot be applied to the general population. Without a known population size, as is the case in a tertiary center study, it is also impossible to compute incidence numbers.

Epidemiological characteristics can help the clinician in formulating a differential diagnosis. As can been seen from our data, age can help make several kinds of orbital malignancies more or less likely, geographical

background on the other hand seems to have no role in discerning between different tumour types because relative frequencies of orbital tumours are comparable in different parts of the world. The incidence of orbital tumours shows a slight relative increase in time, the absolute incidence numbers, however, are still very low, so we do not consider this rising trend to be of clinical importance. The increase of immunocompromised patients may explain this rising trend, for example patients with HIV and solid organ transplant recipients. ${ }^{15}$ In addition, as mentioned by Margo and Mulla more lymphoid hyperplastic lesions are now being diagnosed as lymphoma.

In our study we only used the topographical codes C69.5 and C69.6 to identify orbital tumours. Other codes that can identify tumours located in the orbit are C41.0 for bone tumours of the face and skull and C72.3 for optic nerve tumours. These codes, however, are shared by other cranial bones and optic chiasm or optic tract tumours, respectively, and do not provide enough information to identify primary orbital tumours and were excluded. These side codes, however, were also not used in the incidence studies that are compared with our study in Table 2.

The reliability of the data in our study depends on the completeness of the cancer registration. Estimates of the completeness in registrating cancer cases by the NCR are 96.2 and $98.7 \% .^{16,17}$ Specially trained NCR staff review the full medical record of all patients to minimise the chance of an orbital tumour being part of systemic tumour involvement and thus being non-primary.

In summary, the relative frequencies of histopathological subtypes found in the Netherlands do not differ greatly from the frequencies found in other parts of the world. We also conclude that lymphoma is the most common malignancy of the orbit in the Netherlands. This malignancy is followed by rhabdomyosarcoma, adenocarcinoma, and adenoid cystic carcinoma. The overall average, age standardised incidence, of orbital malignancies is 10 per 10000000 per year and this study shows a slow rise.

Summary

What was known before

- Primary malignant orbital tumour incidence on a population-based level in the United States and Asia.

What this study adds

- Primary malignant orbital tumour incidence on a population-based level in the Netherlands. Also provided is the development of this incidence in time.

\section{Conflict of interest}

The authors declare no conflict of interest.

\section{References}

1 van der Sanden GA, Coebergh JW, Schouten LJ, Visser O, van Leeuwen FE. Cancer incidence in the Netherlands in 1989 and 1990: first results of the nationwide netherlands cancer registry. Coordinating committee for regional cancer registries. Eur J Cancer 1995; 31A: 1822-1829.

2 Margo CE, Mulla ZD. Malignant tumours of the orbit. Analysis of the florida cancer registry. Ophthalmology 1998; 105: 185-190.

3 Lee SB, Au Eong KG, Saw SM, Chan TK, Lee HP. Eye cancer incidence in singapore. $\mathrm{Br}$ J Ophthalmol 2000; 84: 767-770.

4 Cheng CY, Hsu WM. Incidence of eye cancer in taiwan: an 18-year review. Eye 2004; 18: 152-158.

5 Casparie M, Tiebosch AT, Burger G, Blauwgeers H, van de Pol A, van Krieken JH et al. Pathology databanking and biobanking in the netherlands, a central role for PALGA, the nationwide histopathology and cytopathology data network and archive. Cell Oncol 2007; 29: 19-24.

6 Percy C. International Classification of Diseases for Oncology. 3rd ed World Health Organization: Geneva, 2000.

7 'Statistics Netherlands (CBS)'. Population statistics. Available at:http://statline.cbs.nl/StatWeb. Accessed 02/11, 2009.

8 Parkin DM, Muir CS, Whelan SL. Cancer Incidence in Five Continents. Vol III IARC Scientific Publications: Lyon, 1976.

9 Seregard S, Sahlin S. Panorama of orbital space-occupying lesions. The 24-year experience of a referral centre. Acta Ophthalmol Scand 1999; 77: 91-98.

10 Johansen S, Heegaard S, Bogeskov L, Prause JU. Orbital space-occupying lesions in Denmark 1974-1997. Acta Ophthalmol Scand 2000; 78: 547-552.

11 Rootman J. Diseases of the Orbit, A Multidisciplinary Approach. 2nd ed Lippincott Williams \& Wilkins: Philadelphia, 2003.

12 Shields JA, Shields CL, Scartozzi R. Survey of 1264 patients with orbital tumours and simulating lesions: the 2002 montgomery lecture, part 1. Ophthalmology 2004; 111: 997-1008. 
13 Ohtsuka K, Hashimoto M, Suzuki Y. A review of 244 orbital tumours in japanese patients during a 21-year period: origins and locations. Jpn J Ophthalmol 2005; 49 : 49-55.

14 Shikishima K, Kawai K, Kitahara K. Pathological evaluation of orbital tumours in japan: analysis of a large case series and 1379 cases reported in the japanese literature. Clin Experiment Ophthalmol 2006; 34: 239-244.
15 Karcioglu ZA. Orbital Tumors: Diagnosis and Treatment, McNab A. A, The Changing Nature and Behavior of Orbital and Periorbital Tumors. 1st ed. Springer: New York, 2005, pp. 27-33.

16 Berkel J. General practitioners and completeness of cancer registry. J Epidemiol Community Health 1990; 44: 121-124.

17 Schouten LJ, Hoppener P, van den Brandt PA, Knottnerus JA, Jager JJ. Completeness of cancer registration in limburg, the netherlands. Int J Epidemiol 1993; 22: 369-376. 